\title{
Développement durable, paysage, lien social en Seine-Eure (Normandie)
}

\author{
O desenvolvimento sustentável, paisagem, laço social em Seine-Eure (Normandia)
}

Sustainable development, landscape, social link in Seine-Eure (Normandy)

El desarrollo sostenible, paisaje, vínculo social en Seine-Eure (Normandía)

\author{
Nassima Dris* \\ (nassima.dris@univ-rouen.fr)
}

Recebido em 10/06/2015; revisado e aprovado em 07/10/2015; aceito em 07/12/2015

DOI: http://dx.doi.org/10.20435/1984042X2016212

\begin{abstract}
Résumé: Le paysage est un enjeu majeur de la valorisation durable des territoires. Dans cette recherche, la dimension territoriale des faits sociaux est investie sous l'angle du sentiment d'appartenance et d'identité territoriale. Elle interroge des phénomènes sociaux façonnés par des pratiques spatiales individuelles et collectives et par des politiques locales de développement durable.

Mots-clés: paysage; lien social; développement durable.

Resumo: A paisagem é um grande desafio para o desenvolvimento sustentável dos territórios Nesta pesquisa, a dimensão territorial dos fatos sociais é investida em termos de sentimento de pertença e identidade local. Ele questiona fenômenos sociais moldados por práticas espaciais individuais e coletivas e políticas locais de desenvolvimento sustentável .

Palavras-chave: paisagem; ligação social; desenvolvimento sustentável.

Abstract: The landscape is a major challenge for the sustainable development of territories. In this research, the territorial dimension of social facts is vested in terms of the feeling of belonging and local identity. It questions the social phenomena shaped by individual and collective spatial practices and local policies for sustainable development.
\end{abstract}

Key words: landscape; social link; sustainable development.

Resumen: El paisaje es un gran desafío para el desarrollo sostenible de los territorios. En esta investigación, la dimensión territorial de los hechos sociales recae en términos del sentido de pertenencia e identidad local. Cuestiona la forma por las prácticas espaciales individuales y colectivas y las políticas locales de desarrollo sostenible fenómenos sociales.

Palabras clave: paisaje; vínculo social; el desarrollo sostenible.

\section{INTRODUCTION}

Dans les questions territoriales, le paysage est un enjeu majeur dans l'élaboration des programmes de valorisation. La prise en compte de la nature et des paysages dans les projets de développement durable renouvelle les approches sociologiques et anthropologiques des territoires. En effet, les nouvelles configurations sociales et spatiales de plus en plus complexes observées dans une perspective de développement durable amènent à explorer un double processus d'homogénéisation et de différenciation du rapport au territoire. Objet polysémique et transdisciplinaire, le territoire nécessite la mobilisation des approches pragmatiques fondées sur l'expérience du terrain. De ce fait, le territoire apparaît non pas comme un état ou un acquis mais comme " un rythme infini » d'une histoire humaine. D'inspiration Simmelienne, ce mode d'approche aborde le territoire, sa délimitation et son sens par une triangulation exposant l'indigène et son terroir au regard d'autrui (MARIE, 2007, p. 24). Dans cette recherche, la dimension territoriale des faits sociaux est investie sous l'angle à la fois de l'appartenance et de l'altérité. Elle met en évidence des phénomènes sociaux façonnés par des pratiques spatiales individuelles et collectives de l'espace vécu et par des politiques sociales localisées dans des territoires institutionnalisés. Le sentiment d'appartenance à des lieux spécifiques est articulé ainsi aux effets de l'action publique. Il s'agit de mesurer les effets des politiques locales d'aménagement et de valorisation du paysager dans le changement du regard sur les lieux de vie.

\footnotetext{
* Université de Rouen, Normandie, França.
} 


\section{POUR UNE NOUVELLE FORME DE GOUVERNANCE}

Le développement durable apparaît comme une nouvelle forme de gouvernance fondée sur l'équité sociale, l'efficience économique, la protection de l'environnement et la participation citoyenne. Une nouvelle forme de gestion donc, dans laquelle le long terme est valorisé, la dimension du risque introduite et les individus responsabilisés. Mais cette "boîte à outils » facilite-t-elle véritablement l'action ? On notera que de nombreux travaux traitant du développement durable soulignent l'émergence d'une nouvelle utopie à l'instar de l'hygiénisme et du mouvement moderne. S'agit-il alors d'un éternel retour au même? Le développement durable réactive-il les principes de la « table rase » ? Valorise-t-il le retour à des discours purement techniques et politiques pour une " réinvention » des modes d'habiter selon l'adage " apprendre aux gens à habiter »? A ce titre, l'éco-quartier ou l'éco-village s'inscrivent de plus en plus dans une logique compétitive pour asseoir une "vitrine écologique " indispensable aux politiques territoriales. Si sur le plan de l'image, cette logique est porteuse, elle comporte un risque évident de la fabrication des inégalités et des ségrégations socio-spatiales. Il convient de souligner que le territoire et son corollaire, la territorialité relèvent bel et bien de la logique de l'habiter et de la temporalité dans des contextes spécifiques confrontés à un processus de renouvellement constant et donc, à une réinvention permanente du quotidien. Aussi, les situations actuelles appellent à reconsidérer la célèbre notion du « droit à la ville» (LEFEBVRE, 1968). L'explosion urbaine à l'échelle planétaire impose la réactualisation du «droit à la ville » et de la même façon, le droit à un environnement de qualité pour les populations les plus vulnérables. En effet, le développement durable soulève une question fondamentale, celle de " la justice environnementale » en termes de droit à un environnement de qualité pour tous. Il s'agit de l'accessibilité à un environnement de qualité des populations défavorisées qui ne bénéficient pas d'une visibilité sur « l'ardoise environnementale» (BLANC, 2012). Si la problématique du développement durable soulève celle de l'équité sociale c'est parce que le rapport à l'environnement n'est pas fait d'événements exceptionnels mais d'activités ordinaires inscrites dans la vie quotidienne.

Or, certaines initiatives se réclamant du « développement durable » sont considérées, le plus souvent, soit comme une "nouvelle idéologie ", soit comme une reformulation de politiques anciennes. Il n'en reste pas moins que le "développement durable » fait référence à des " espaces intégrés » nécessitant une approche globale tant sur le plan épistémologique que pratique même si la question du « développement durable » comme outil opérationnel reste entière.

La recomposition perpétuelle des formes sociales et spatiales met à mal l'idée selon laquelle le territoire serait le résultat exclusif d'un processus d'urbanisation même si l'urbanisme s'inscrit indéniablement dans un projet global de société. Cela est d'autant plus vrai que la vie sociale laisse remonter à la surface ce que le projet urbain en a exclu. C'est pourquoi en matière d'aménagement, le "désordre " peut être considéré comme une ressource dont le bon usage permet d'écarter certaines parties aveugles de l'action urbaine. La culture locale et le sentiment d'appartenance $n^{\prime}$ ont de sens que lorsque les liens de proximité sont significatifs et que les individus ont véritablement une existence sociale. Ce constat amène à envisager les actions de développement local durable dans une démarche interactive mettant à contribution les populations. Le projet territorial devient, à ce titre, un instrument d'affirmation et/ou de restauration d'un système de valeurs lié à une "identité locale partagée ». Or, les moyens engagés dans des actions de désenclavement de certaines catégories sociales ne suffisent pas à enrayer ou du moins à réduire les fragmentations qui, en perdurant, confirment non seulement le morcellement du système de référence mais aussi les risques permanents de cloisonnement des différences et paradoxalement, une marginalisation progressive des valeurs locales.

Mais, peut-on considérer que le changement d'échelle peut, à lui seul, changer les politiques (NEGRIER, 2005), et permet-il $\mathrm{d}$ 'identifier la nature du lien que les acteurs de la politique publique entretiennent avec 
le territoire ? C'est ainsi que les initiatives se réclamant $\mathrm{du}$ " développement durable » sont présentées comme étant le plus souvent, une réaffirmation de politiques anciennes sur la préservation du capital naturel. Cela interroge les enjeux d'une gestion négociée du développement local et l'implication des citoyens dans des processus qui les concernent durablement.

\section{LE PAYSAGE, UN RÉFÉRENTIEL EN QUESTION}

Questionner les rapports de l'Homme à son environnement naturel invite à considérer les nouvelles manières d'habiter entre espace bâti et espace naturel. La démarche se fonde sur l'appréciation collective de la nature et les formes d'appropriation diversifiées ainsi que les enjeux d'une mise en scène du paysage. Il s'agit de mettre en lumière l'expérience sensible des individus et l'émergence d'un sentiment collectif dans un « jeu social associant savoirs, réflexivité et communication $»$. Si le citadin dans sa relation à l'environnement, construit et expose " une créativité ordinaire » (RANCIERE, 2000), ce système d'interactions orientant les représentations du monde serait-il une voie nouvelle pour apprécier la dimension sociale du développement durable? C'est par un processus permanent de transformation et d'adaptations réciproques que l'homme interagit avec la nature en lui donnant du sens (DEWEY, 2012), celle-ci étant à la fois un objet concret et une réalité représentée. C'est ainsi que l'expérience ordinaire des individus $\mathrm{s}^{\prime}$ appuie à la fois sur la permanence d'un monde ancré dans l'espace et dans le temps et la contingence comme donnée essentielle pour comprendre la dynamique du rapport à la nature.

En renaissance perpétuelle, la nature est faite de transition, de mouvement, de rythme. Il en est de même pour les interactions entre l'homme et la nature qui $\mathrm{n}^{\prime}$ ont rien d'un acquis définitif mais restent marquées par des fusions, des incertitudes et des tensions. Les " corythmes entre Nature et Culture constituent l'enjeu des reliances régénératrices des milieux urbains » (PAQUOT; YOUNÈS, 2010, p. 43). En effet, les analyses anthropologiques de l'espace mettent en lumière la relation étroite entre culture et nature dans la construction de l'espace vécu et la symbolique qui s'y rattache. Il s'agit d'un processus résidentiel articulant différents modes d'habiter humain à l'expérience de la nature.

Le regain d'intérêt pour le paysage tant dans les discours et les pratiques que dans les projets territoriaux s'appuie sur l'élargissement de la notion à des dimensions fondamentales liées au rapport à l'environnement. Il s'agit de la pérennité des liens forts des hommes avec la nature : « $\mathrm{Si}$ la notion de paysage mérite d'être honorée, ce n'est pas seulement parce qu'elle se situe, de manière exemplaire, à l'entrecroisement de la nature et de la culture, des hasards de la création et de l'univers et du travail des hommes, ce n'est pas seulement parce qu' elle vaut pour l'espace rural et pour l'espace urbain. C'est essentiellement parce qu'elle nous rappelle que cette terre, la nôtre, que nos pays sont à regarder, à retrouver qu'ils doivent s'accorder à notre chair, gorger nos sens, répondre de la façon la plus harmonieuse qui soit à notre attente. » (SANSOT, 1983).

La notion de " paysage » se situe entre une lecture des interactions multiples du milieu naturel et des sociétés, d'une part et sa mise en tension par un regard historiquement et socialement construit, d'autre part (CLOAREC; COLOMB; KALAORA, 1989).

La polysémique de la notion englobe des interprétations diverses liées aux constructions sociales, aux représentations, aux émotions, à l'esthétique, etc. Aussi, l' « appréciation des paysages » ou la " consommation paysagère » peuvent être envisagées dans une perspective privilégiant l'interprétation du goût dans laquelle l'éminence est accordée à la maîtrise des codes renforçant les mécanismes de la distinction sociale (SERFATY-GARZON, 1991). De ce fait, le paysage $n^{\prime}$ est pas seulement une représentation ou un espace concret support d'émotions ou d'actions pratiques, il est tout à la fois un objet réel, construit et représenté dont la variabilité de sens résulte de la tension des facteurs sociaux auxquels le paysage est soumis. Le rapport à l'environnement est ici analysé à partir des activités humaines en perpétuelle recomposition. La perspective socio-anthropologique del'espace considère la 
relation au paysage et donc à l'environnement immédiat comme une construction sociale de l'espace habité (LYNCH, 1969; LEDRUT, 1968; SANSOT, 1983).

Si aujourd'hui, l'expression « paysage » fait référence à un idéal de "bien-être ", le rapport à la nature n'est pas dénué de tensions, de peurs et d'incertitudes. Si nous considérons que c'est dans la double fonction du territoire creuset phénoménologique et cadre cognitif pour l'action (FABUREL, 2010) que les enjeux environnementaux s'inscrivent et puisent leur sens, l'instrumentalisation de la nature à des fins politiques réduit la portée de la demande sociale. Reste à explorer comment observer la pluralité des territoires, des niveaux de décision, des usages et des attentes des populations souvent contradictoires ? Comment les habitants interprètent-ils ou réinterprètent-ils le paysage et quelles sont leurs attentes en la matière ? Par quels systèmes d'acteurs et d'action, le paysage et la nature deviennent-ils des outils du développement durable?

\section{SPÉCIFICITÉS ET DÉLIMITATION DU TERRAIN D'ENQUÊTE}

La Communauté d'Agglomération Seine-Eure $(\mathrm{CASE})^{1}$ a connu depuis sa création en 2001, une longue phase de construction. C'est en juillet 1993 que le

\footnotetext{
${ }^{1}$ Le terrain de recherche s'étend sur l'ensemble de la communauté d'agglomération. Les investigations (observations, entretiens, passation du questionnaire...) ont été menées de manière plus précise sur certaines communes du fait de leur représentativité, notamment en termes de patrimoine et de paysage. L'enquête de terrain s'appuie sur le discours des acteurs, une observation ethnographique et un questionnaire composé essentiellement de questions ouvertes. Sur cette base, trois entités paysagères ont été retenues ainsi que deux à trois communes par entités: Les Boucles de Seine avec les communes de Léry (2095 hab.), Poses (1 143 hab.) et Amfreville sous les Monts (555 hab.) ; Les Plateaux agricoles avec les communes de La Haye Malherbe (1 493 hab.) et Le Mesnil Jourdain (249 hab.) ; Les Vallées de l'Eure et de l'Iton avec les communes d'Acquigny (1 669 hab.) et Le Vaudreuil (3599 hab.) ; Les trois pôles urbains avec la ville nouvelle de Val de Reuil (13 775 hab.) ; la ville historique de Louviers (18 635 hab.) et Pont de l'Arche, une ville entre Seine et Eure (4 044 hab.).
}

Programme d'Aménagement Concerté du Territoire (PACT) a été lancé pour apporter une dynamique nouvelle à un territoire aux caractéristiques à la fois industrielles, urbaines et sociales. En 1997, le Syndicat Intercommunal Val de Seine donnera naissance à la Communauté de Communes regroupant Louviers, Incarville et Val de Reuil. Puis, les lois relatives à l'orientation et l'aménagement durable des territoires promulguées en 1999 sous le nom de « Pays » (loi dite Voynet) donneront une nouvelle dynamique au regroupement de communes.

Il en sera de même pour les lois relatives à la coopération intercommunale mettant en place un cadre juridique, institutionnel et financier pour la création de Communautés de Communes Urbaines et d'Agglomérations (loi dite Chevènement sur l'intercommunalité). Cependant, le seuil de 50.000 habitants nécessaire à la formation d'une communauté de communes, est devenu l'enjeu majeur des élus porteurs de projet. Cetteloi a étéle point de départ d'un important travail de mobilisation des communes visant la formation en 2001 de deux regroupements intercommunaux : Communauté de Communes Seine-Forêt de Bord et Communauté d'Agglomération Seine-Eure.

Les indicateurs sociaux en 2006-2007 signalent une forte proportion d'ouvriers, un taux de chômage élevé, un niveau de formation plutôt bas et une part importante de logements sociaux. La proportion d'ouvriers $(36,58 \%)$ est nettement supérieure à la moyenne nationale $(24,39 \%)$ et à la moyenne en Haute Normandie (30,18\%). Cependant, le revenu fiscal médian est légèrement inférieur au revenu national. La CASE compte 30,50\% de logements sociaux en 2007, soit le taux le plus élevé de la région Haute Normandie, qui elle-même a un taux supérieur à la moyenne nationale, soit $20,8 \%$ contre $14,8 \%$.

Le logement social est un des indicateurs parmi les plus significatifs du «profil social » de l'agglomération. Si le nombre élevé de maisons $(68,4 \%)$ confirme surtout la dominance de la maison individuelle dans le monde rural, il renseigne aussi sur le choix urbanistique du pôle urbain de Val de Reuil qui se caractérise d'une part, par une structure du logement social sur dalles et d'autre part, par un important dispositif de lotissements 
en maisons individuelles autour du noyau ancien de la ville nouvelle. La fourchette de « 10 ans et plus » indiquant l'ancienneté dans le logement n'est pas éloignée des deux autres niveaux, soit $47,3 \%$ contre $48,6 \%$ pour la Haute Normandie et $48,2 \%$ pour l'ensemble de la France.

Sur le plan démographique et sociospatial, la CASE présente des caractéristiques spécifiques qui la distinguent de l'ensemble de la Région Haute-Normandie (INSEE, 2007). Sa densité est la plus élevée de la Région soit $235,1 \mathrm{hab} / \mathrm{km}^{2}$. Avec un taux de $29,6 \%$ des moins de 20 ans, la population de la CASE est en moyenne, plus jeune que celle de Haute-Normandie $(26,6 \%)$ et de la France métropolitaine $(24,6 \%)$.

En 2006, la population active de 15 à 64 ans, domiciliée dans la Communauté d'Agglomération, est ouvrière à $36,5 \%$ contre 30,18 \% pour la Région et $24,39 \%$ pour le niveau national. Cette forte proportion d'ouvriers est liée à l'implantation sur le territoire d'un secteur industriel important, notamment l'industrie pharmaceutique (Aventis Pasteur, Janssen Cilag). En 2007, les inactifs représentaient $28,9 \%$ de la population ayant entre 15 et 64 ans, alors que $30 \%$ seulement des employés du secteur pharmaceutique résidaient sur le territoire. Les trois pôles urbains (Louviers, Val de Reuil et Pont de l'Arche) concentrent plus de la moitié de la population du territoire avec une forte proportion d'ouvriers et un faible niveau d'éducation.

La configuration spatiale du territoire se distingue par trois niveaux significatifs du processus d'urbanisation : - des entités urbaines denses et structurées ; des bourgs ayant un «certain niveau d'équipement »; des villages ayant conservé une "structure traditionnelle ». Le territoire présente ainsi des caractéristiques singulières entre villes et campagne pouvant le différencier d'autres territoires normands et renforcer son pouvoir attractif. Toutefois, l'hétérogénéité spatiale comporte des risques de segmentation entre les principaux pôles économiques et le reste de l'agglomération (MUNICIPALITE SERVICE, 2006).

Elle implique par ailleurs une hétérogénéité sociale pouvant expliquer un contexte politique mouvementé (THIARD,
2004). Pourtant en 2003, l'INSEE indiquait une " mixité sociale relativement avancée » (INSEE, 2003). En effet, si certaines communes sont marquées par une forte présence de populations aux revenus modestes, elles sont relativement peu nombreuses par rapport à l'étendue du territoire. Néanmoins, la moitié des communes dispose d'un quota significatif de logements sociaux.

Les Boucles de Seine ont été classées dans la catégorie «Espaces Environnementaux Sensibles " selon différents niveaux de protection (SCOT, 2010). Les « espaces naturels indice $1 »$ sont les plus remarquables par leur «étendue, leur richesse et leur représentativité » tels que les terrasses alluviales de la Seine classées Natura 2000. Ces dernières s'étendent sur les Boucles de Seine amont et plus spécifiquement sur la carrière du Plessis à Amfreville sous les Monts, le Lac du Mesnil et les îles de la Seine à Poses. Cette zone est d'importance internationale pour l'hivernage et la migration des oiseaux.

Les « espaces naturels indice 2 » regroupent les zones rurales interstitiels représentées par les coteaux calcaires d'Amfreville sous les Monts, les mares et les plans d'eau de la réserve de la Grande Noë. Quant aux « corridors écologiques », ils couvrent les passages reliant des espaces naturels permettant aux animaux et aux plantes de migrer. Ces passages se situent entre le Bois de Pîtres et la Côte des Deux Amants, entre la Côte des Deux Amants et les îles de Poses, entre le Secteur de Haute Villette et les Coteaux de Vironvay, entre les îles de Seine, le Bois de Saint Martin et le Bois Fleuret sur les communes de Connelles, Tournedos et Herqueville. Les mesures de protection de ces espaces naturels ont pour objectifs d'atténuer les risques dus à l'impact néfaste de l'homme sur la transformation des milieux naturels. Les plateaux agricoles connaissent une pression foncière croissante (Ecoparcs, urbanisation, infrastructures routières...), des problèmes de ruissellement et d'érosion des sols touchant les parcelles agricoles des communes de Surtauville, La Haye-Malherbe... (AREHN, 2003).

Cette zone est qualifiée par "une sensibilité paysagère accrue " (SCOT, 2010, p. 13) sur le rebord des plateaux. La Haye Malherbe et le Mesnil-Jourdain se rapprochent 
de l'entité définie par la SCOT sous le nom du "Plateau du Neubourg, Val Eure et Iton ", classée en indice 1. D'autres zones comme celle du Bois du Mesnil-Jourdain sont classées « corridors écologiques ».

L'axe Seine / Eure / Iton réunit des espaces naturels vitaux pour le territoire urbain comme « la trame bleue du SCOT». Toutefois, les berges connaissent des conflits d'usages divers liés à l'extraction de matériaux, l'urbanisation le long des berges et les risques de pollution menaçant les ressources en eau. Alors que l'entrée de la ville d'Acquigny est identifiée comme étant «sensible » en termes de patrimoine naturel et de cadre de vie (SCOT, 2010, p. 7), le Bois d'Acquigny fait partie des « espaces interstitiels » (espaces naturels indice 2) constituant le maillage naturel du territoire, tout comme la vallée de la Seine et de l'Iton et leurs vallées secondaires. Il en est de même pour le vallon du Becdal, classé pour sa faune et sa flore. Le village du Vaudreuil est quant à lui, situé dans une boucle de la Seine, entre la forêt de Bord et le fleuve. Ce village est traversé par l'Eure et se situe au centre de la voie verte qui s'étend sur $13 \mathrm{~km}$ d'Incarville à Poses. La commune est concernée par la préservation des axes urbains présentant une ambiance paysagère qualitative (CITADIA, 2008, p. 51). Elle dispose d'un Golf placé sur un site d'une grande qualité paysagère et historique avec une grange du XVIIe siècle.

Le pôle urbain le plus doté $d^{\prime}$ environnement naturel est la ville nouvelle de Val de Reuil qui occupe une superficie de 2800 hectares dont 1370 d'espaces verts (ETIENNE; GIRARD, 2007). Bordée par un long chemin de randonnée longeant les rives de l'Eure, et bénéficiant au nord-ouest de la forêt de Bord, le site de Val de Reuil est d'une qualité paysagère riche et diversifiée. Il est de surcroit bien desservi par les transports en commun. Les espaces naturels sont variés et la végétation (arbres, parcs, pergolas) est omniprésente dans le Germe de ville. En effet, la ville bénéficie de nombreux espaces verts : les squares François Mitterrand et la Commune de Paris, le jardin de la Grosse Borne... La réserve ornithologique de la Grande Noë constitue également, un des attraits de la ville. La principale caractéristique de Val de Reuil est son urbanisme de dalle qui a conduit à la séparation des flux (piétons et voitures) sur deux niveaux reliés par des rampes et des escaliers.

\section{L'ENTRE-DEUX TERRITORIAL, ENTRE VILLE ET CAMPAGNE}

La rencontre de la ville et de la campagne donne au paysage naturel, une visibilité plus grande. Il devient facilement identifiable et accessible au point de fonctionner parfois comme un vecteur du lien social. Toutefois, les disparités sociales, économiques, patrimoniales et historiques peuvent constituer un frein à l'équilibre territorial malgré la mobilisation des habitants autour de certaines questions sensibles. Les configurations spatiales notamment le découpage administratif, mais aussi le transport public ne facilitent pas le rapprochement entre les différentes parties du territoire. Dès lors, la transversalité des projets indispensable à l'intercommunalité connaît quelques lenteurs comportant les risques d'une inégalité de traitement au sein $\mathrm{du}$ territoire et la marginalisation de certaines communes, notamment les plus « petites ».

Pourtant, la concertation impliquant des acteurs sociaux n'est pas totalement absente, elle se réalise dans certains cas, comme par exemple, lors du débat sur l'étendue et les limites du territoire de la communauté d'agglomération. Ce débat a mis en lumière les problèmes auxquels est confrontée la communauté d'Agglomération comme le désenclavement de certaines communes situées aux limites administratives du territoire et l'étalement urbain considéré comme source de dégradation de l'espace rural, de détérioration des sites protégés, de réduction des terres agricoles et de disparition des paysages emblématiques de la région. Cela résume une situation de tensions permanentes entre l'urbanisation rampante, la volonté de protection des villages et des terres agricoles mais aussi les risques d'un déséquilibre territorial. Il s'agit surtout de la menace qui pèse sur les territoires ruraux $d u$ fait de l'hégémonie des villes et des rapports de pouvoir entre les pôles urbains.

Plus globalement, ces conflits autour de la préservation de l'espace rural renforcent la nécessité de valoriser le patrimoine 
naturel comme enjeu économique mais aussi identitaire. Les requêtes à propos des pistes cyclables, l'aménagement de voies vertes et de chemins de randonnée sont fréquentes. Ces aménagements sont perçus par les habitants comme étant favorables à la préservation de l'environnement et au développement du lien social. Pourtant, de nombreuses initiatives en faveur d'un partenariat diversifié à l'intérieur et à l'extérieur du territoire mettent en interaction plusieurs structures : des associations de préservation des milieux naturels, culturels et du patrimoine, la Fondation du Patrimoine et les Compagnons du Patrimoine, le Service Régional de l'Inventaire (SRI), le Conservatoire des Sites Naturels de Haute Normandie (CSNHN), l'Agence Régionale de l'Environnement de Haute Normandie (AREHN) et autres partenaires. Mais les rivalités entre les élus n'encouragent pas la participation de la population et affaiblissent l'exercice de la démocratie locale. Dans ce contexte, les actions sont souvent fragmentées ou inadaptées comme par exemple la difficile accessibilité aux équipements culturels ou encore, une politique de formation éloignée de l'offre d'emploi des secteurs d'activité implantés sur le territoire.

Toutefois, l'association entre patrimoine naturel et développement durable fait consensus. Dans une logique de transmission et de solidarité intergénérationnelle, ces deux notions participent au même processus qui consiste à articuler le temps présent des sociétés, à leur passé et leur avenir. Le " profil de développement durable » de la CASE élaboré par l'Agence Régional de l'Environnement de Haute-Normandie (2003), pointe " un déficit d'image » dû, en grande partie, aux effets de l'urbanisation de ces dernières années. Il n'est donc pas aisé de faire converger les préoccupations paysagères vers des objectifs précis du développement durable par la traduction du discours en action.

Or, la restauration del'image du territoire passe par la mise en place de diverses actions y compris une communication spécifique, destinée aux élus et au grand public, de même qu'un large réseau de partenaires à l'échelle de la Région. Notre enquête menée sur plusieurs communes a montré le manque d'informations, particulièrement dans le domaine environnemental et cela, malgré le nombre important d'associations locales et une politique $d^{\prime}<$ ? $>$. Le dispositif mis en place cible en priorité une communication à portée générale éloignée $\mathrm{du}$ profil $\mathrm{du}$ territoire. Cela se traduit chez les habitants, par une méconnaissance du fonctionnement institutionnel $\mathrm{du}$ territoire et un manque d'informations sur les questions sociales (logement, emploi, formation, éducation, santé, mobilité et culture). Cela renvoie à l'idée selon laquelle la « restauration du lien social » passe par le développement social durable des territoires. Or, si les dispositifs de l'Agenda 21 s'appuient sur des phases de concertation avec les habitants et les acteurs sociaux, la participation des habitants ne semble pas très significative du fait de leur faible présence lors des débats publics.

\section{LE PAYSAGE, LIEU DE SOCIABILITÉ ?}

Le «portrait identitaire » d'un territoire permet d'identifier les signes qui le caractérisent comme la culture, le patrimoine architectural ou paysager, les savoir-faire ou encore les personnalités emblématiques qui y vivent. La Normandie est considérée comme " un territoire d'une extrême diversité renforcée par une puissante image locale » : 75 types de paysages sont recensés avec une richesse exceptionnelle de l'architecture dans chacun des « pays » de Normandie mais aussi les patois ou les accents qu'on peut entendre, la faune, la flore, etc. « Même les pommiers et l'armoire normande sont différents suivant les terroirs !... Ici, les gens ne sont pas de Normandie mais d'une Normandie !» ${ }^{2}$.

Sur la base d'un questionnaire adressé à 28 communes du territoire, une nomenclature des lieux du patrimoine local spécifiant un patrimoine paysager de dimension remarquable (plateaux, coteaux et vallées) et un patrimoine plus modeste décrit comme « paysage de contact » où cohabitent différentes entités naturelles et historiques (pelouses calcaires et industrie de l'Eure) a été élaborée (HABITAT ET DEVELOPPEMENT, 2004). Grâce à un réseau multimodal permettant

\footnotetext{
${ }^{2}$ Entretien rapporté par Co-Managing, bureau conseil pour le marketing territorial et touristique.
} 
de passer d'une ambiance à une autre, l'accès aux sites naturels est aisé sur le territoire. La nature est donc très présente dans les pratiques quotidiennes ou occasionnelles. Aux initiatives individuelles de pratiques de paysage s'ajoutent diverses propositions émanant des associations, des services culturels et des équipes pédagogiques spécialisées dans la faune et la flore. Ces initiatives visent à sensibiliser le public à la protection de la nature et à un comportement adapté. Au regard de l'instabilité des milieux nécessitant une attention particulière, certains interlocuteurs considèrent que la nature se suffit à elle-même et ne nécessite pas de transformation pour pouvoir en profiter. Pourtant, l'aménagement de la voie verte $(13 \mathrm{~km})$ est perçu par les usagers comme un espace d'où il est possible d'observer une variété de fleurs et d'animaux le long de l'Eure.

Ce site connaît un mode doux de transport, un aménagement adapté sur terrain plat permettant une accessibilité sécurisée (sans voiture) et une esthétique environnementale appréciable (végétation, bords de l'Eure, présence d'animaux...). Toutefois, ce site majoritairement appréciée connaît certaines carences. En effet, les bords de l'Eure sont pointées par les habitants comme étant insuffisamment sécurisés et pouvant représenter un danger pour les enfants. Il en est de même pour d'autres sites comme le lac du Mesnil dont les abords nécessitent un entretien substantiel. Certains risques pèsent donc sur cet environnement naturel malgré les aménagements (voies goudronnées, tunnels pour piétons et passages aménagés pour animaux).

Le paysage perçu et vécu comme lieu de sociabilité force à reconsidérer le rapport à la nature au-delà de la vitrine écologique des sites remarquables. Les habitants de la CASE désignent le patrimoine naturel (sites remarquables, jardins de proximité, parcs naturels) comme étant plus visible, plus proche et plus accessible que le patrimoine bâti. Pour faire image, la base de loisirs Léry-Poses dont la configuration paysagère parfaitement intégrée au site au point de la confondre avec un état naturel, est clairement identifiée comme une nature de proximité, à la fois sauvage et domestiquée donc sans risques majeurs pour les usagers. Dans ce cas de figure, le paysage est soumis à une adaptation permanente, une façon de maintenir l'aspect général du territoire dans un environnement naturaliste.

Le paysage associé au développement durable prend une forme intermédiaire entre représentations et mode de développement. La valorisation du paysage en tant que ressource, traduit l'émergence d'un mode de développement spécifique fondé sur la durabilité territoriale par un renouvellement des ressources. Dans cette perspective, la diversité et la richesse des paysages invitent à établir des priorités dans la mise en œuvre d'actions adaptées. Or, la hiérarchisation des priorités n'est pas toujours aisée. Les entretiens menés sur le territoire pointent l'absence de critères singuliers pour affirmer une identité normande même si ces derniers ne sont rien d'autres que des stéréotypes de la Normandie :

- « tout est beau et gentil mais il n'y a pas d'élément fort »;

- « la Normandie imaginaire, c'est celle du Pays de Caux avec les pommiers en fleurs, c'est Giverny, Les Andelys... » ;

- « Certains pensent même que la Normandie ce n'est pas ici !».

Face à ce manque réel ou imaginaire, le premier atout du territoire est d'ordre pratique à savoir, une position géographique facilitant la mobilité :

- « en quelques dizaines de minutes, on atteint la vallée de l'Andelle, de l'Iton, Giverny, Harcourt et le champ de bataille, Abbaye Bec-Hellouin, Rouen et Paris ».

Toutefois, ce critère ne suffit pour dynamiser l'attractivité :

- « le territoire Seine-Eure est au centre de plein de choses mais pas un centre en soi »

Pour contourner cette image de « simple lieu de passage », le territoire s'efforce de composer avec ses limites en palliant les effets néfastes de l'industrialisation par le potentiel paysager des vallées de l'Eure et de l'Iton. Il en est de même du rapprochement ville/ campagne pouvant peser sur l'attractivité du territoire. Dès son origine, la ville nouvelle de Val de Reuil a été conçue comme " une ville à la campagne ». Aujourd'hui, elle s'oriente vers l'implantation d'un secteur industriel respectueux de l'environnement. Le territoire 
dans son ensemble s'inscrit de fait dans l'entredeux : la ville et la campagne, l'industrie et de l'agriculture, le patrimoine naturel et le patrimoine bâti, le développement durable et le tourisme, etc.

Le lien avec la nature repose aussi sur l'expérience vécue des jardins ouvriers. A l'origine, les jardins dits " ouvriers " faisaient partie de la politique d'habitat social dans laquelle ils jouaient un rôle d'ajustement et de recadrage de l'espace urbain. Il s'agissait pour les municipalités de l'époque d'éviter qu'une réserve foncière ne se transforme en "zone » attirant une animation indésirable dans des terrains vagues. Dans ce contexte, l'aménagement des jardins ouvriers permettait de "se décerner à moindre frais un prix de paternalisme et un satisfecit toujours politiquement utile. » (SERFATY-GARZON, 1991). Au-delà des préoccupations foncières et urbanistiques, les jardins ouvriers soutiennent un processus d'intériorisation d'une identité et d'un statut d'une catégorie sociale déterminés par des instances politiques. Aujourd'hui, la dénomination « jardins familiaux ${ }^{3}$ plus valorisante, correspond à un élargissement de la demande de parcelles à d'autres catégories professionnelles de l'habitat social. La publicisation des jardins familiaux a abouti à une uniformisation du lieu selon un ordre défini par les services de la municipalité.

Or, la conception d'un jardin ne répond pas des règles préconçues mais relève $d^{\prime} u n$ arrangement permanent avec le lieu et ses composantes. Le rapport à la nature consiste à « hybrider le public et le privé, l'individuel et le collectif, l'engagement et le détachement. [...] C'est imaginer, non pas un rapport social déterminé (une fonction de détente, de loisir, de sport ou autre), mais une sociabilité potentielle qui, dans et par le jardin, aura lieu ou n'aura pas lieu. » (ZEPF, 2004, p. 37). Les liens que les gens entretiennent avec leur environnement (naturel ou bâti) mettent en lumière à la fois, la «culture des gens » spécifiée par la fluidité des valeurs en perpétuelle évolution et le sentiment d'appartenance associé à l'existence sociale des individus.

\footnotetext{
${ }^{3}$ Cette dénomination a été retenue par la loi $\mathrm{n}^{\circ}$ 52-895 du 26 juillet 1952 portant « Codification de la législation des jardins familiaux ».
}

\section{CONCLUSION}

Le paysage facilite-t-il le lien social ? Peut-t-il jouer le rôle de « ciment identitaire » ? Les réponses à ces questions ne peuvent être que partielles ou spécifiques à des situations singulières. $C^{\prime}$ est en ce sens que la complexité des réalités sociales incite à penser avec ses pieds, en arpentant littéralement un territoire dans ses différentes dimensions pour découvrir les relations qui se nouent en chaque lieu (CALAME, 2005). Par les effets de la concertation, cette proximité permet d'échapper à un sentiment de relégation ou de dépréciation. La spécificité du territoire de la CASE réside dans sa bipolarité urbaine et sa diversité sociale et paysagère. Comme ailleurs, les tensions entre l'urbanisation et la préservation des paysages sont récurrentes. Toutefois, le territoire se démarque par une configuration spatiale de "l'entre-ville » (SIEVERTS, 2004) se situant à l'interface du rural et de l'urbain. A mi-chemin entre la ville et la campagne, entre les réseaux de communication locaux et mondiaux, «l'entreville » est à la fois, espace de production et de consommation. Elle permet le développement de contacts de proximité et d'appropriation de l'environnement immédiat. Il s'agit d'une approche mêlant développement durable, paysage, architecture et urbanisme en faveur d'une reconversion économique par l'intégration des territoires à la fois urbains et ruraux. Dans ce cas de figure, l'intérêt porte sur les points de rencontre et d'articulation par lesquels les urbains défendent la pérennité des usages agricoles et les ruraux ne nient pas l'urbanité (BAYLE, 2011). L'enjeu majeur pour le territoire de la CASE reste la préservation des terres agricoles comme ressources naturelles essentielles même si le paysage est désigné comme la principale valeur en termes d'images et de bien-être. Toutefois, la proximité des terres agricoles et l'engouement pour les jardins de proximité participent-il à un changement progressif vers de nouvelles manières $d^{\prime}$ habiter le paysage?

\section{BIBLIOGRAPHIE}

AGENCE REGIONALE DE L'ENVIRONNEMENT DE HAUTE-NORMANDIE (AREHN). Profil de développement durable de la CASE, décembre 2003. 
BAYLE, Christophe. Les lisières, territoires d'innovation pour le Grand Paris. Métropolitiques, 2011.

BLANC, Nathalie. Les nouvelles esthétiques urbaines. Paris: Armand Colin, 2012.

CALAME, Pierre. Territoires. Penser localement pour agir globalement. Paris: Editions Charles Léopold Mayer, 2005.

CITADIA. Retour sur les enjeux du diagnostic territorial. Paris, 2008.

CLOAREC, Jacques; COLOMB Gérard; KALAORA, Bernard. Crise du paysage? Ethnologie française (3), $\mathrm{t}$. 19, p. 197-200, 1989.

DEWEY, John. Expérience et nature. Paris: Gallimard, 2012.

DRIS, Nassima (Dir.). Patrimoines et développement durable: Ressources - Enjeux - Lien social. Rennes: Presses universitaires de Rennes, 2012.

DRIS, Nassima (Dir.). Territoires et territorialité. Regards pluridisciplinaires. Paris: L'Harmattan, 2007.

DRIS, Nassima. Patrimoine et développement local: l'appropriation collective du patrimoine comme forme d'intégration sociale. Interações, Campo Grande, MS, v. 8, n. 13, p. 9-18, set. 2006.

ÉTIENNE, Claire; GIRARD, Julie. Ville nouvelle de Val-de-Reuil. Un patrimoine en devenir, Histoire urbaine (20), p. 77-100, 2007.

FABUREL, Guillaume. Des mots de l'environnement aux maux des territoires. In: PAQUOT, T.; CHRIS, Y. Philosophie de l'environnement et milieux urbains. Paris: La Découverte, 2010.

HABITAT ET DEVELOPPEMENT. Etude de définition d'une politique d'aide à la préservation et à la mise en valeur des patrimoines bâti et naturel, diagnostic, Avril 2004.

INSEE. Haute-Normandie et CASE, «SCOT Seine Eure Forêt de Bord - Synthèses locales », 2003.

. «CA Seine-Eure, Chiffres clés », dossier thématique, 2007.

LEDRUT, Raymond. L'Espace social de la ville. Paris: Anthropos, 1968.
LEFEBVRE, Henri. Le Droit à la ville. Paris: Anthropos, 1968.

LYNCH, Kevin. L'image de la cité. Paris: Dunod, 1969.

MARIE, Michel. De l'urbaniste à l'anthropologue: question de territoire. In: DRIS, N.; Territoires et territorialité. Regards pluridisciplinaires, innovations et societes. Paris: L'Harmattan, 2007. p. 15-26.

MUNICIPALITE SERVICE. Diagnostic du Plan de Déplacement Urbain, octobre 2006, p. 52.

NEGRIER, Emmanuel. La question métropolitaine. Les politiques à l'épreuve du changement d'échelle territoriale. Grenoble: Presse universitaire de Grenoble, 2005.

PAQUOT, Thierry; YOUNES, Chris. Philosophie de l'environnement et milieux urbains, Paris: La Découverte, 2010.

RANCIÈRE, Jacques. Le partage du sensible. Esthétique et politique, Paris: La Fabrique, 2000.

SANSOT, Pierre. Variations paysagères. Paris: Klincksiek, 1983.

SCHEMA DE COHERENCE TERRITORIALE (SCOT). Seine-Eure Forêt de Bord - Synthèses locales, décembre 2003, p. 6.

. Seine-Eure-Forêt de Bord, Présentation du Document d'Orientations Générales, Le Bipôle - Axe structurant, 2008.

Seine-Eure Forêt de Bord. La prise en compte de l'environnement, 2010.

SERFATY-GARZON, Perla. La ville et ses restes. In : GERMAIN, A. (Dir.). L'aménagement urbain. Promesses et défis. Montréal : Institut Québécois de Recherche sur la Culture, 1991. p. 232-267.

SIEVERTS, Thomas. Entre-ville, une lecture de la Zwischenstadt. Marseille: Editions Parenthèses, 2004.

THIARD, Philippe. Val de Reuil et Louviers: deux sociétés urbaines locales pour un projet ou la difficile justification sociale du contrat d'agglomération Seine-Eure, communication, UMR-ESO, Rennes 21-22 octobre 2004.

ZEPF, Marcus. Concerter, gouverner et concevoir les espaces publics urbains. Lausanne, Suíça: Presses Polytechniques et Universitaires Romandes, 2004. 